call it $g^{\prime}$ distinct from $e$. Let $U$ be an open three-cell subset of $R$ which includes $e$. Let $N$ be a two-sphere made up of elements of $G$. The existence of arbitrarily small two-spheres of this kind is proved as above by choosing the element $g$ sufficiently near to $e$, and we may assume that $N$ is in $U$. We may also assume that $L N$ is in $U$ and that $g^{\prime} N$ is outside $N$ : as must be the case if $N$ is small enough.

The arc $L$ may now be used to define a deformation of $N$ to $g^{\prime} N$. Under this deformation all points swept out by $N$ are in $G$. Furthermore every point inside $N$ is swept out by the deformation. Hence every point of $R$ inside $N$ is in the group $G$. The group $G$ is thus seen to contain open subsets and, because of homogeneity, $G$ is open in $R$. It must therefore coincide with $R$. The assumption that a proper subgroup $G$ was transitive on $S$ has now led to a contradiction, and the proof is therefore complete.

Smith College and

Queens College

\title{
ON ORDERED ALGEBRAS ${ }^{1}$
}

\section{A. A. ALBERT}

In his first Madison Colloquium lecture M. H. Stone connected the theory of convex bodies with linear sets over an ordered field. It was natural then to ask whether his theory could be extended by replacing these fields by ordered rings and indeed to ask whether there exist ordered rings which are not fields. I discussed this question at that time with S. MacLane and we attempted to answer it. MacLane has since found an example, ${ }^{2}$ in the literature, of a noncommutative ordered quasi-field. It is not an algebra (of finite order) however and it is my purpose in this note to give a very brief proof in elementary language of the following decisive result.

THEOREM. Every ordered algebra is a field.

We first observe some known consequences of the order postulates. ${ }^{3}$ The postulates on products imply that an ordered ring contains no divisors of zero and hence that every ordered algebra is a division algebra $D$. Then $D$ has a unity quantity $1=1^{2}>0$, the sums

1 Presented to the Society, December 2, 1939.

${ }^{2}$ Cf. Reidemeister, Grundlagen der Geometrie, p. 40. It is also shown in this text that archimedian ordered quasi-fields are fields.

${ }^{3}$ The order postulates on page 40 of my Modern Higher Algebra were called postulates for an ordered field but are valid for arbitrary rings. 
$1+1+\cdots+1$ are all positive, $D$ is a normal division algebra of degree $n$ over a non-modular field $K$, its centrum. If $u \neq 0$ is in $D$, then $u u^{-1}=1>0, u$ and $u^{-1}$ are either both positive or both negative, the transforms $u d u^{-1}$ of every positive quantity of $D$ are all positive.

Let then $n>1$ so that $D$ contains a quantity $b$ not in its centrum $K$. The minimum function of $b$ is $g(x)=x^{n}+a_{1} x^{n-1}+\cdots+a_{n}$ with $n>1$ and the $a_{i}$ in $K$. Since $K$ is non-modular, $D$ contains $b-m^{-1} a_{1}$ and its negative. One of these is a positive quantity $d$ of $D$ with minimum function $h(y)=y^{m}+b_{2} y^{m-2}+\cdots+b_{m}$ for $b_{i}$ in $K$. By a theorem of Wedderburn ${ }^{4} h(y)=\left(y-d_{m}\right) \cdots\left(y-d_{1}\right)$ for transforms $d_{i}$ of $d$, the $d_{i}>0$, their sum is $d_{1}+\cdots+d_{m}=0$ by our choice above. This contradicts our hypothesis that $D$ is ordered. Hence $n=1, D=K$ is a field.

In more technical terms our proof is simply the remark that, if it exists, a noncommutative ordered division algebra contains a positive quantity $d$ of zero trace, whereas this trace is a sum of transforms of $d$ and must be positive.

\section{University of Chicago}

\footnotetext{
${ }^{4}$ See the proof of Theorem 3, p. 230, of L. E. Dickson's Algebras and their Arithmetics.
} 